\title{
Selective Excitation on Tip-Enhanced Raman Spectroscopy by Pulse Shaping Femtosecond Laser
}

\author{
Yingjie Xia ${ }^{1,2,3} \cdot$ Hua Zhao ${ }^{1,4} \cdot$ Chunjie Zheng ${ }^{1} \cdot$ Shian Zhang ${ }^{1} \cdot$ Donghai Feng ${ }^{1} \cdot$ Zhenrong Sun $^{1} \cdot$ Tianqing Jia $^{1}$
}

Received: 24 April 2018 / Accepted: 10 August 2018 / Published online: 29 August 2018

(C) The Author(s) 2018

\begin{abstract}
In this paper, we propose a scheme for achieving ultrafast coherent control of the selective excitation among three excited states in stimulated Raman scattering process on a tip-enhanced Raman spectroscopy (TERS). The center frequencies of the pump and Stokes laser pulses are $14,000 \mathrm{~cm}^{-1}$ and $12,500 \mathrm{~cm}^{-1}$, and their spectral bandwidths are both $700 \mathrm{~cm}^{-1}$. By properly modulating the spectral phase distribution and cutting the frequency components, the stimulated Raman transition probabilities of two excited state keep maximal, while the other one can be suppressed to zero. The shaped pump and Stokes pulse irradiate obliquely into the TERS nanostructure containing a single layer molecule. The impulse response in temporal and frequency domain is calculated by using finite-difference time-domain (FDTD) simulation followed by Fourier transform. The frequency components and the relative phase are same with the corresponding input pulses, but the intensities are enhanced by more than 10 times. Compared with the case without the TERS nanostructure, the probability of selective excited Raman transition increases by more than 4 orders of magnitude, and the selective depressed Raman peak keeps at 0.
\end{abstract}

Keywords Tip-enhanced Raman spectroscopy · Single molecule detecting · Ultrafast coherent control · Pulse shaping

\section{Introduction}

Tip-enhanced Raman spectroscopy (TERS) is a powerful technique resulting in strongly increasing Raman signals from molecules attached to a metallic nanostructure. It is of great significance in many fields such as chemistry, biology, medicine, pharmacology, and environmental science [1-14]. TERS is one of the useful tools for detecting single molecule with high sensitivity and molecular specificity. R. Zhang et al. reported Raman spectral imaging and resolved the inner

Tianqing Jia

tqjia@phy.ecnu.edu.cn

1 State Key Laboratory of Precision Spectroscopy, College of Physics and Materials, East China Normal University, Shanghai 200062, People's Republic of China

2 School of Information Science and Engineering, University of Jinan, Jinan 250022, People's Republic of China

3 Shandong Provincial Key Laboratory of Network Based Intelligent Computing, University of Jinan, Jinan 250022, People's Republic of China

4 Department of Mathematics and Physics, Shanghai Dianji University, Shanghai 200240, People's Republic of China structure and surface configuration of a single $\mathrm{H}_{2}$ TBPP molecule [10]. Dmitry Kurouski et al. compared the nanoscale redox behavior of Nile Blue by electrochemical TERS with conventional cyclic voltammetry [15]. To gain insight into intramolecular coupling, substrate coupling, and dynamic processes, Kyoung-Duck Park et al. used TERS at variable and cryogenic temperatures, to slow and control the motion of a single molecule [16]. Besides, Raman spectroscopy of single molecule was used to study molecular isomerization and its intermediate processes [17].

How to enhance the signal-noise ratio is a key problem in detecting single molecule by TERS, which is very important in improving the spatial resolution and expanding its applications in the fields of molecular dynamics and controlling [10-12, 15, 16]. A main method to enhance the signal-noise ratio is the tip fabrication $[1,2,18]$. Recently, $\mathrm{R}$. Zhang et al. demonstrated chemical mapping of a single molecule with a spatial resolution less than $1 \mathrm{~nm}$ through the generation of an efficient double-resonance enhancement for both Raman excitation and Raman emission by precisely adjusting the tunneling gap [10]. However, several neighboring Raman peaks were excited simultaneously, which depressed the signal-noise ratio of the TERS spectra.

An effective method for the coherent control of Raman scattering is femtosecond pulse shaping technique, where the 
laser spectral phase and/or amplitude were modulated to achieve the constructive/destructive interference between different path integral leading to expected/depressed outcomes [19-27]. This method was widely used in controlling molecular vibration state, rotation state, ionization state, isomerization, and other molecule dynamics. By tailoring the phase of probe pulse, Dan Oron et al. demonstrated a narrow-band coherent anti-Stokes Raman spectroscopy (CARS) signal with a width of less than $15 \mathrm{~cm}^{-1}$, which is an order of magnitude narrower than the signal from a transform limited (TL) pulse [19]. They further eliminated the non-resonant CARS background and selectively excited one of two neighboring Raman levels by shaping both the pump and the Stokes pulses with an appropriate spectral phase function [20]. Selective excitation of CARS by shaping femtosecond laser pulses using adaptive learning algorithms, good signal-to-background ratio, and high sensitivity were realized in many kinds of molecules [21-23].

Spatiotemporal control of optical fields in plasmonic nanostructures excited by shaped ultrafast laser pulse was studied theoretically [28-33]. Mark Stockman et al. predicted and theoretically investigated the coherent control of nanoscale localization of ultrafast optical excitation in V-shaped nanostructures by spectral phase modulation, which permits one to coherently control the local light fields to be concentrated at a desired location at certain times [29, 30]. Laser pulse shaping techniques were further used to spatiotemporal control of optical fields in nanoantennas and plasmonic circuits [32]. Using finite-difference time-domain (FDTD) simulations followed by Fourier transformation, the impulse response of a nanostructure in the time and frequency domain was obtained. Ultrafast dynamics of nanoplasmonic dephasing of individual silver nanoparticles and small clusters were studied via accurately delayed femtosecond laser pulses, which provided insight into inhomogeneous and homogeneous broadening mechanisms in nanoplasmonic spectroscopy [34]. The response over a broad spectrum of a snowflake antenna was used, inversely, in femtosecond pulse shaping [28]. Spatiotemporal control of optical fields in plasmonic nanostructures was widely applied in the fields of high-harmonic and single attosecond pulse generation $[35,36]$, ultrafast coherent surface enhanced Raman scattering [37, 38], and vibrational wave packet motion on single molecules [39].

In this paper, we introduce a scheme for achieving a precise control of the selective excitation among three excited states in the stimulated Raman scattering process by shaping femtosecond laser pulses. In our model, two excited states are maximally populated, while the other one is suppressed to zero by phase modulating and frequency cutting of the pump and Stokes pulse. The shaped pulses are transformed into time domain by inverse Fourier transform, and the temporal evolution at the probe point of the gap center of TERS structure is calculated by using COMSOL Multiphysics. The temporal, phase, and frequency distributions of the response pulse are calculated and discussed respectively. The theoretical results show that the enhanced and selected Raman scattering is realized simultaneously in the TERS structure by shaping the pump and Stokes femtosecond laser pulses.

\section{TERS Nanostructure and Simulation Method}

The TERS nanostructure, as depicted in Fig. 1, consists of a silver conical tip over an Ag film [4]. The tip is a spherical crown with radius $r=10.0 \mathrm{~nm}$, the bottom radius $R=39.1 \mathrm{~nm}$ with a full cone angle of $40^{\circ}$. The tip-film distance $d$ is $2.0 \mathrm{~nm}$. The silver film is set as $150 \times 150 \times$ $60 \mathrm{~nm}^{3}$, with a complex permittivity, $\varepsilon$, taken from [40], and a permeability of $\mu=1$. The TERS spectra of large molecules, such as Rhodamine 6G, malachite, porphyrin, and DNA, were widely studied $[2,7,10,12,13,15-18$, 41-43]. The real part of the refractive index of these materials is mostly in the range of $1.5-1.7$, and single molecular layer is of $0.5-0.6 \mathrm{~nm}$ thick. In the TERS nanostructure, a single molecular layer of $0.5 \mathrm{~nm}$ thick is covered on the whole silver film. The real and imaginary part of the refractive index are set as 1.6 and 0.1 , respectively. Stokes and pump lasers are both p-polarized and radiate collinearly on the TERS nanostructure along an angle of $\theta=30^{\circ}$. The origin of coordinates is set at the center of the upper surface of the molecular layer.

The finite element method (COMSOL Multiphysics) is employed to calculate the optical properties of the TERS nanostructure. The simulation region is $750 \times 750 \times$ $750 \mathrm{~nm}^{3}$. A cubical perfectly matched layer is used to absorb all outgoing radiation and to eliminate the reflections at the domain boundaries. During the numerical simulation, the minimum element size of local adaptive mesh refinement is set as $0.5 \mathrm{~nm}$.

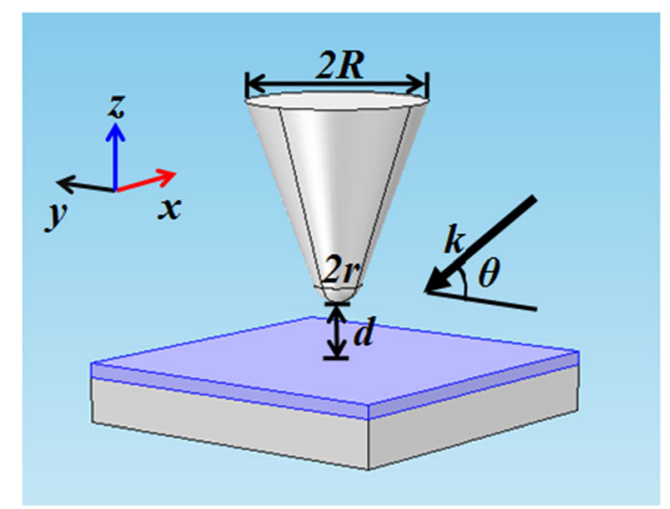

Fig. 1 Schematic diagram of TERS nanostructure and the incident light, where the blue film is the single molecular layer 


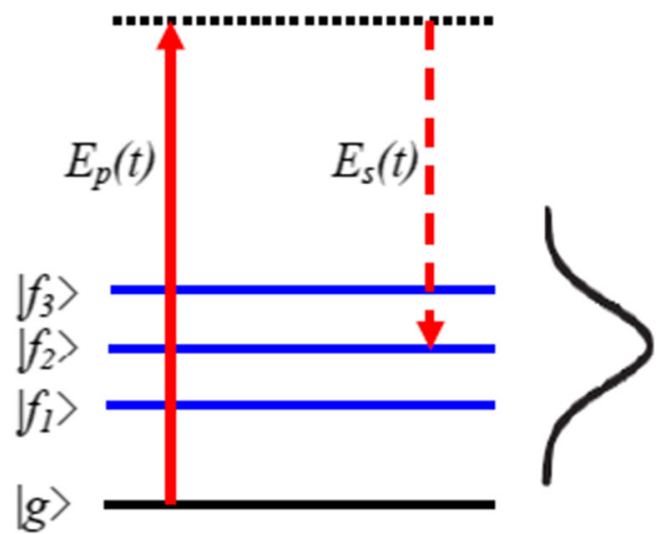

Fig. 2 Schematic diagram of the stimulated Raman transition process. The three finial states are all excited simultaneously by the pump field $E_{p}(t)$ and Stokes field $E_{s}(t)$

\section{Coherent Control and Selective Excitation of Raman Transitions by Shaping Femtosecond Pulses}

Coherent control was intensely studied in the application of selective excitation of Raman scattering by shaping the ultrafast pump and Stokes laser pulses [19-27]. In the following, we theoretically study how to achieve a high degree selective excitation of two Raman peaks among three excited states.

Figure 2 shows a four-level atomic system and the stimulated Raman transition process. $\mid g>$ and $\mid f_{i}>(i=1,2,3)$ are the ground and final excited states, respectively. Two ultrafast laser fields, the pump field $E_{p}(t)$ and the Stokes field $E_{s}(t)$, irradiate on the atomic system and induce the stimulated Raman transition $|g>\rightarrow| f_{i}>$ [44]. Assuming the population is initially in the ground state $|g\rangle$, the Raman transition probability of the excited states $\mid f_{i}>$ can be expressed by $[44,45]$.

$P_{f_{i}}=\left|a_{f_{i}}\right|^{2} \propto\left|\int_{0}^{+\infty} E_{p}\left(\Omega_{p}\right) E_{s}^{*}\left(\Omega_{p}-\omega_{f i}\right) d \Omega_{p}\right|^{2}$

where $\omega_{f i}$ is the transition frequency from the ground state $|g\rangle$ to the final excited states $\mid f_{i}>$. $E_{p}\left(\Omega_{p}\right)$ and $E_{s}\left(\Omega_{s}\right)$ are, respectively, the Fourier transform of $E_{p}(t)$ and $E_{s}(t)$ given by

$$
\begin{aligned}
& \mathrm{E}_{\mathrm{p}}\left(\Omega_{\mathrm{p}}\right)=\mathrm{E}_{\mathrm{p} 0} \exp \left[-\left(\Omega_{\mathrm{p}}-\Gamma_{p}\right)^{2} / \delta_{p}^{2}\right] \exp \left[i \Phi_{p}\left(\Omega_{\mathrm{p}}\right)\right] \\
& \mathrm{E}_{\mathrm{s}}\left(\Omega_{\mathrm{s}}\right)=\mathrm{E}_{\mathrm{s} 0} \exp \left[-\left(\Omega_{\mathrm{s}}-\Gamma_{s}\right)^{2} / \delta_{s}^{2}\right] \exp \left[i \Phi_{s}\left(\Omega_{\mathrm{s}}\right)\right]
\end{aligned}
$$

where $\Gamma_{p}$ and $\Gamma_{s}$ are the center frequency, and $\delta_{p}$ and $\delta_{s}$ are the full width at half maximum (FWHM) of the pump and Stokes pulse spectra, respectively.

The TERS spectra of Rhodamine 6G, malachite, porphyrin, and DNA were intensely studied $[2,7,10,12,13$, 15-18, 41-43]. There are many Raman peaks in the range of $1000-1700 \mathrm{~cm}^{-1}$. In our theoretical model, three Raman peaks of $1600 \mathrm{~cm}^{-1}, 1520 \mathrm{~cm}^{-1}$, and $1400 \mathrm{~cm}^{-1}$ are studied as representative sample. The center frequencies of the pump and Stokes laser pulses $\Gamma_{p}$ and $\Gamma_{s}$ are set at $14000 \mathrm{~cm}^{-1}$ and $12,500 \mathrm{~cm}^{-1}$, and the FWHM $\delta_{p}$ and $\delta_{s}$ are both $700 \mathrm{~cm}^{-1}$. The difference between the center frequencies of the pump and stokes lasers is nearly equal to the average value of three Raman peaks, and the bandwidths are much larger than the differences among the three Raman peaks, which ensure the three Raman modes are all efficiently excited.

Spectral phase and amplitude modulations were used to improve the Raman spectral resolution, selective excitation, or to enhance the transition probability $[44,46]$. In this paper, we design a modulation composed of rectangular phase modulation and rectangular tailoring the Stokes laser spectrum, as shown in Fig. 3a. The frequency components in the window with a width of $240 \mathrm{~cm}^{-1}$ are preserved and those out of the window are cutoff. At the meantime, a rectangular $\pi$ phase modulation with a width of $80 \mathrm{~cm}^{-1}$ is applied on the central part of the frequency component, while others keep at 0 phase.

After pulse shaping as mentioned above, the temporal distribution is obtained by inverse Fourier transform as

$E(t)=\frac{1}{2 \pi} \int_{-\infty}^{+\infty} E(\Omega) e^{i \Omega t} d \Omega$

Figure $3 \mathrm{~b}$ shows the temporal distribution of the electric field intensity of the Stokes laser after the modulation. The laser pulse changes from a Gaussian shape into a complex
Fig. 3 Schematic diagram of the modulations of the Stokes field. a Laser pulse with a modulation composed of rectangular phase modulation (blue line) and rectangular tailoring the spectrum (red shadow). b Temporal distribution of the electric field intensity of the Stokes laser after the modulation
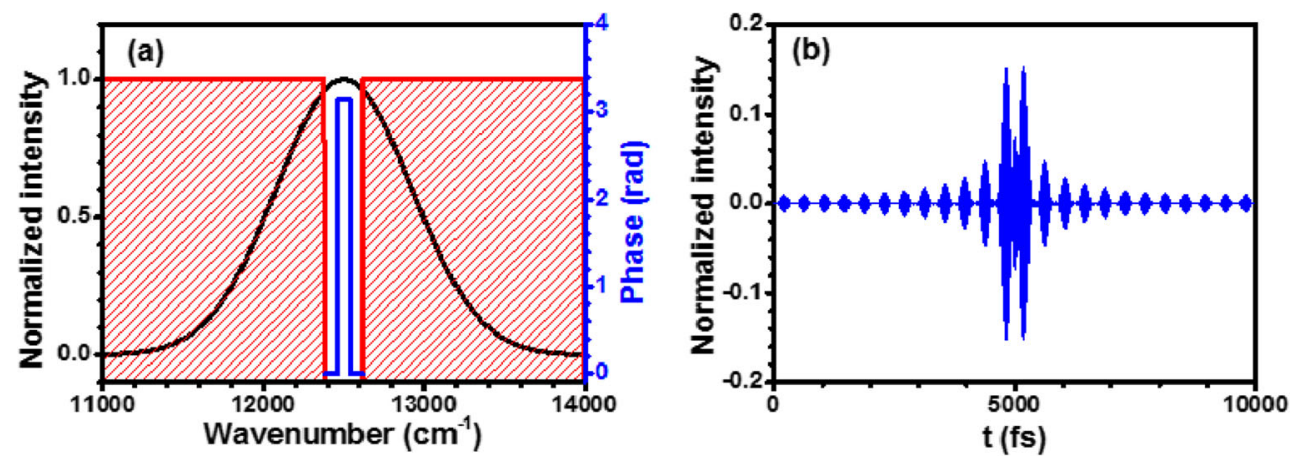

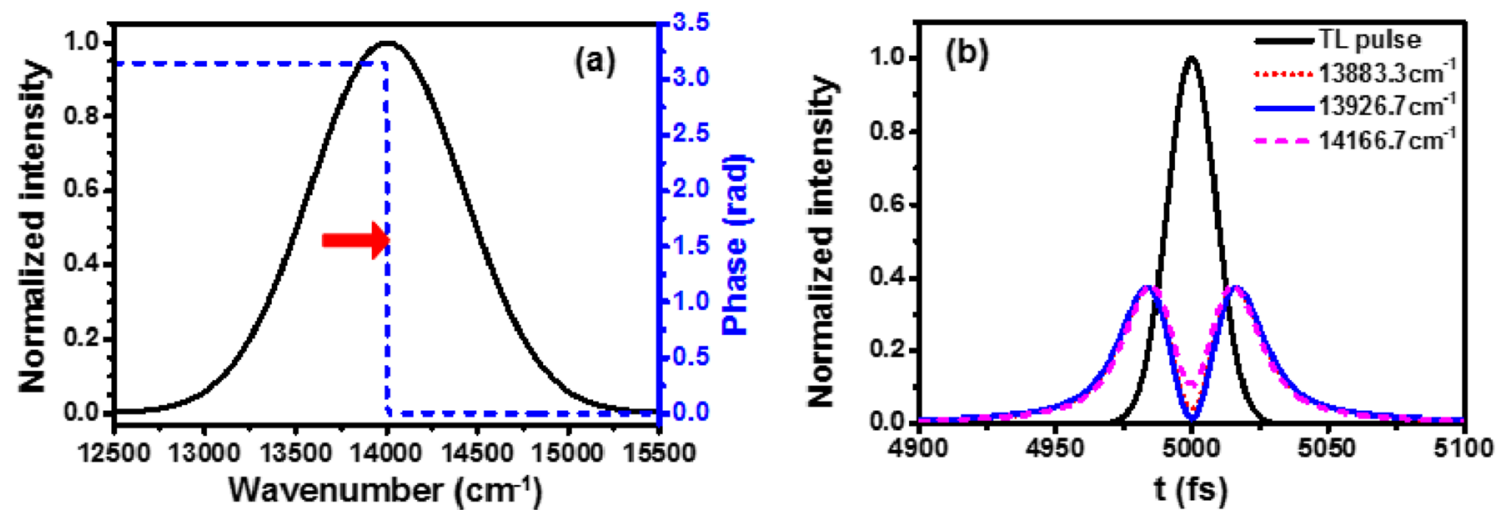

Fig. 4 Schematic diagram of the modulations of the pump field. a The pump laser pulse with a $\pi$ phase step (blue dash line). b Temporal distribution of the pump laser intensity with a $\pi$ phase modulation at different step position. The black line is the initial TL pulse

profile with many small peaks caused by the amplitude and phase modulation. The temporal FWHM of the laser intensity elongates to $480 \mathrm{fs}$ with three dumbbell-shaped peaks. The maximum value of the laser intensity is greatly depressed, only 0.023 of the initial Gaussian pulse.

Figure 4a presents the $\pi$ phase step modulation of the pump laser spectrum, which indicates that the spectral phase is $\pi$ for all frequencies smaller than the phase step position, and is 0 for larger frequencies. By $\pi$ step scanning from low to high frequency, the TL Guassian pulse transforms into a saddletype profile, as shown in Fig. 4b. The peak value of laser intensity as the step position at $13883.3 \mathrm{~cm}^{-1}$ is reduced to $37.7 \%$ of the initial TL pulse. The temporal distribution of laser intensity as the step positions at $13926.7 \mathrm{~cm}^{-1}$ and $14,166.7 \mathrm{~cm}^{-1}$ are also shown in Fig. $4 \mathrm{~b}$.

The stimulated Raman transition probability as a function of the $\pi$ phase step position of pump laser is calculated and depicted in Fig. 5, where the Stokes pulse shaping keeps as that shown in Fig. 3a, b. When the step position is lower than $13,767 \mathrm{~cm}^{-1}$, the probabilities of three Raman transition are all same as the values of TL pump pulse. This is because the wave number in these frequency domains is too small and cannot take part in the Raman processes, as shown in Eq. (1). The cases are same as the step position is larger than $14,200 \mathrm{~cm}^{-1}$.

With further increasing $\pi$ phase step position, the Raman transition pf3 begins to be suppressed, while pf1 and pf 2 keep the same value. When $\pi$ phase step position increases to $13,883.3 \mathrm{~cm}^{-1}$, Raman transitions 1 and 2 are well excited and the probabilities pfl and pf 2 are same as the values for the pump laser being a TL pulse. However, pf3 is suppressed to zero, which is resulted from the destructive interference among different optical pathways in the stimulated Raman transition process.

When the step position scans to $13,926.7 \mathrm{~cm}^{-1}$ and $14,166.7 \mathrm{~cm}^{-1}$, the transition probabilities of pf2 and pf1 are selected suppressed to zero, respectively. Moreover, the transition probability pf3 is larger than the case of TL pump laser by $37 \%$ as the step position at $13926.7 \mathrm{~cm}^{-1}$, which is caused by the constructive interference among optical pathways excited by the shaped Stokes laser and pump laser.

The influence of the width of the rectangular $\pi$ phase modulation on the selection excitation of Raman transition is further studied. The calculation results indicate that if the rectangular $\pi$ phase modulation width of the Stokes laser, as shown in Fig. 3a, is larger than $80 \mathrm{~cm}^{-1}$, the Raman peak pf3 can be thoroughly depressed, but the other two cannot be depressed to zero. If the modulation width is larger than $120 \mathrm{~cm}^{-1}$, none of the three Raman peaks can be depressed to zero. If the modulation width is less $80 \mathrm{~cm}^{-1}$, all of the three Raman peaks can be depressed to zero. However, as one Raman peak is depressed to zero, the probabilities of the other two become smaller. These results indicate that the pulse shaping shown in Fig. 3 is an effective method to realize selective excitation of two Raman transitions among three ones.

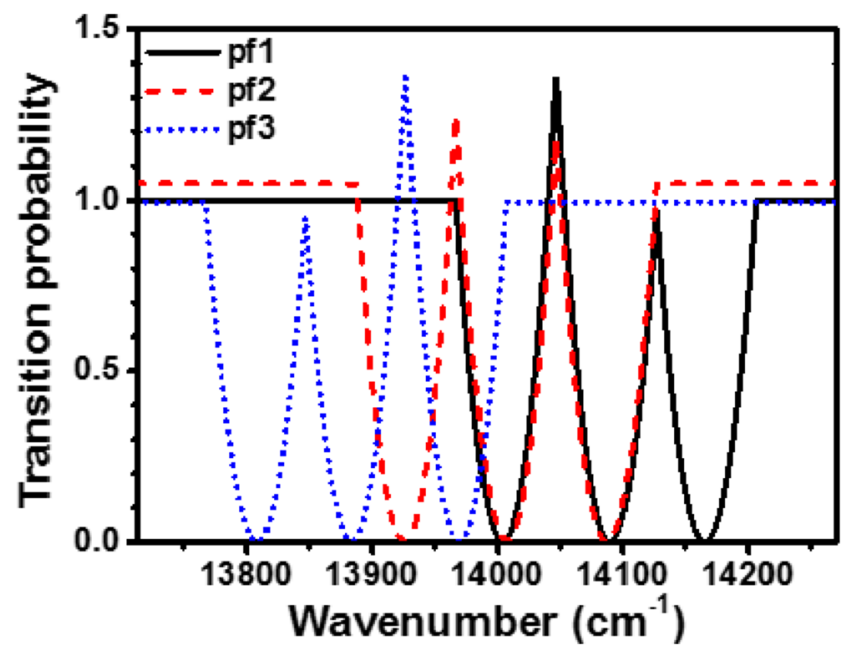

Fig. 5 The stimulated Raman transition probability as the function of the $\pi$ phase step positions of pump laser, where pfl for the TL pump pulse is set as 1 

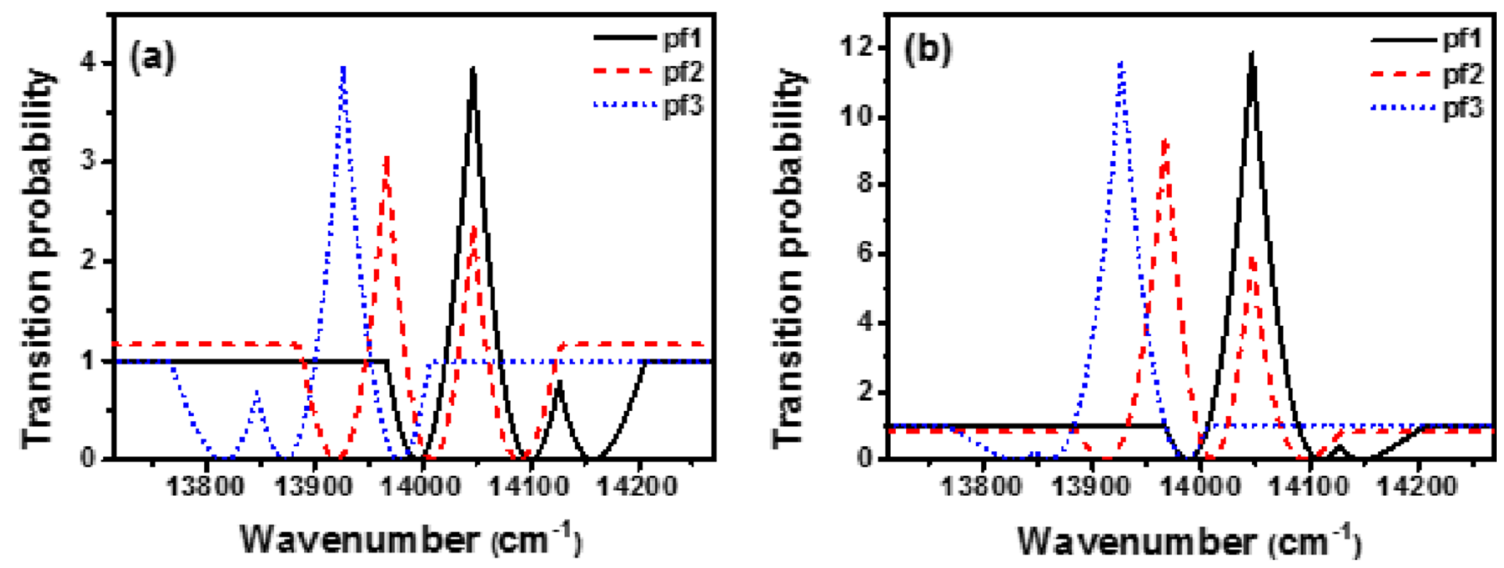

Fig. 6 The stimulated Raman transition probability for $\mathbf{a}$ the FWHM is $300 \mathrm{~cm}^{-1}$ and $\mathbf{b} 200 \mathrm{~cm}^{-1}$, where pfl for the TL pump pulse is set as 1

The FWHM of pump and Stokes laser pulses are both $700 \mathrm{~cm}^{-1}$, much larger than the gap between the Raman peaks at $1400 \mathrm{~cm}^{-1}$ and $1600 \mathrm{~cm}^{-1}$. The influence of FWHM on the selection excitation of Raman transition is further studied. Figure $6 \mathrm{a}, \mathrm{b}$ shows the stimulated Raman transition probability for the FWHM of pump and Stokes laser pulses are $300 \mathrm{~cm}^{-1}$ and $200 \mathrm{~cm}^{-1}$, respectively. When $\pi$ phase step position scans to $13,816.7 \mathrm{~cm}^{-1}$, Raman transitions 1 and 2 are well excited and the probabilities pf1 and pf 2 are the same as the values for the pump laser being a TL pulse (Fig. 6a). However, pf3 is depressed to zero, which is resulted from the destructive interference among different optical pathways in the stimulated Raman transition. When the step position scans to $13,920.0 \mathrm{~cm}^{-1}$ and $14,156.7 \mathrm{~cm}^{-1}$, the transition probabilities of pf2 and pfl are selective depressed to zero, respectively. Moreover, the transition probability $\mathrm{pf} 1 / \mathrm{pf} 2 / \mathrm{pf} 3$ enhances by $3.95 / 3.08 / 3.97$ times as the step position at 14046.7/13966.7/ $13926.7 \mathrm{~cm}^{-1}$, which is caused by the constructive interference among optical pathways excited by the shaped Stokes and pump pulses. The case for FWHM of $200 \mathrm{~cm}^{-1}$ is similar with the cases of $700 \mathrm{~cm}^{-1}$ and $300 \mathrm{~cm}^{-1}$, except the higher transition probability of $\mathrm{pf} 1 / \mathrm{pf} 2 / \mathrm{pf} 3$. The transition probability of $\mathrm{pf1} / \mathrm{pf} 2 / \mathrm{pf} 3$ enhances by $11.8 / 9.46 / 11.6$ times as the step

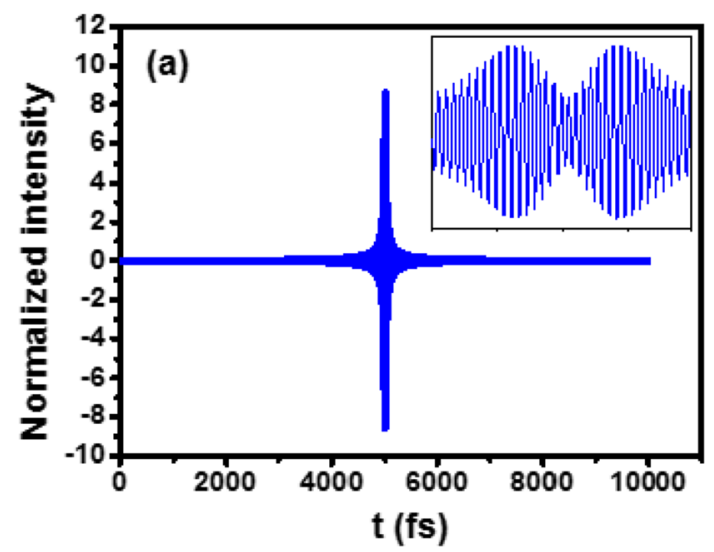

position at $14046.7 / 13966.7 / 13926.7 \mathrm{~cm}^{-1}$. For narrower FWHM of the pump pulse, the destructive effect due to the rectangular modulation of Stokes pulse is larger. Therefore, the constructive interference caused by the $\pi$ phase modulation of the pump pulse is stronger. These results indicate that the scenario of selective excitation of Raman transitions is suitable for femtosecond laser pulse with different FWHM.

\section{Ultrafast Coherent Control of TERS}

When $\pi$ phase step position of pump pulse is at $13883.3 \mathrm{~cm}^{-1}$, Raman transitions pfl and pf2 are well excited, while pf3 is depressed to zero. The temporal distribution of the pump and Stokes pulse electric field is shown in Figs. 3 and 4. The impulse response of the TERS structure to the shaped pump and Stokes pulse in temporal and frequency domain are obtained by using FDTD simulation followed by Fourier transform. The simulation time region is set as $[0,10,000] \mathrm{fs}$, and the interval is $0.1 \mathrm{fs}$.

Figure 7 exhibits time-dependent intensity of the real part of the electric field, where the probe point is at the gap center. The output pump pulse is enhanced by more than 14 times,

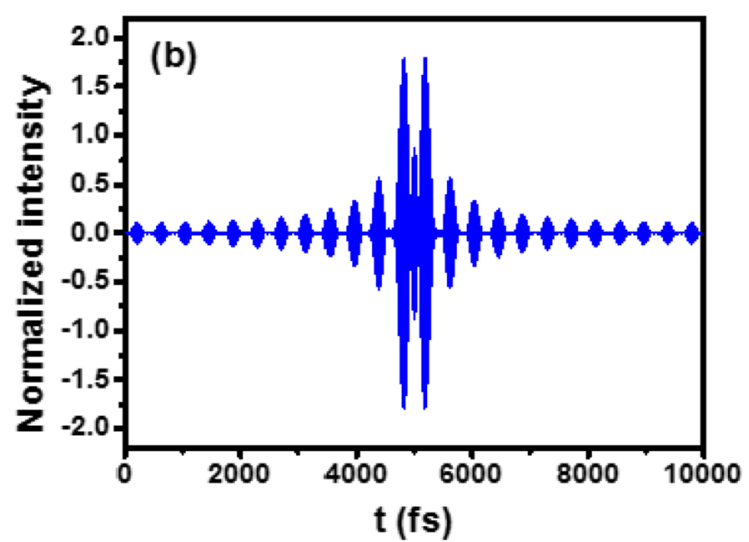

Fig. 7 Time-dependent intensity of the real part of the electric field of a pump pulse and $\mathbf{b}$ Stokes pulse, where the inset in $\mathbf{a}$ is the enlarged view of the central part of $80 \mathrm{fs}$ 

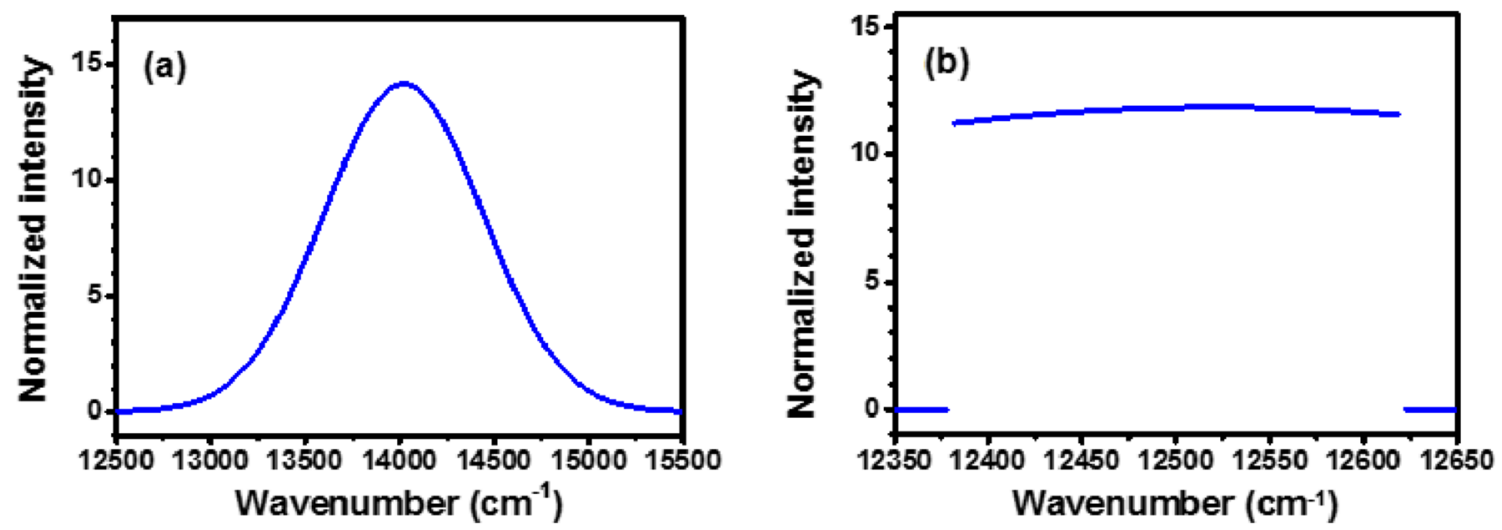

Fig. 8 Frequency distribution of $\mathbf{a}$ the output pump pulse and $\mathbf{b}$ the Stokes pulse

while keeping the similar profile with the input pulse. The result is normalized by the electric filed intensity of the Gaussian laser pulse before phase and amplitude shaping. The electric field of the Stokes pulse is also increased by more than 12 times. We calculated the scattering spectrum, charge distribution, and electric field of the TERS nanostructure. The result shows that the field enhancement in the gap is due to the non-resonant lightning rod effect.

The frequency distribution of the output pulses is obtained by Fourier Transformation as

$E(\Omega)=\int_{-\infty}^{+\infty} E(t) e^{-i \Omega t} d t$

Figure 8 shows the frequency distribution of the pump and Stokes pulse. The frequency components are same with the corresponding input pulses, but the intensities are enhanced by more than 14.0 and 11.5 times, respectively.

The phase of the impulse response is calculated according the formula [47].

$\Phi_{\text {impulse }}(\Omega)=\arg \left[\frac{E_{\text {output }}(\Omega)}{E_{\text {input }}(\Omega)}\right]=\Phi_{\text {output }}(\Omega)-\Phi_{\text {input }}(\Omega)$

where $\Phi_{\text {output }}(\Omega)$ is the phase of the corresponding response of TERS structure, i.e., the phase of the output of the pump

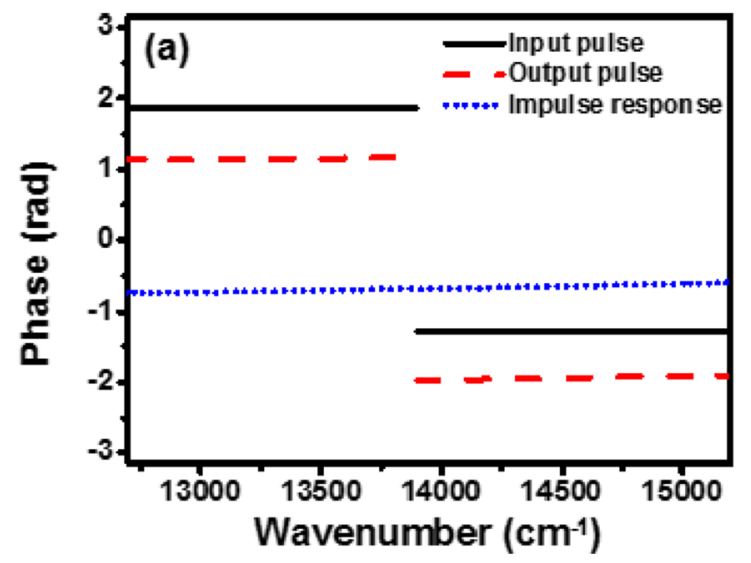

field or the Stokes field, and $\Phi_{\text {input }}(\Omega)$ is the phase of the source field, i.e., the phase of the input of the pump field or the Stokes field after shaping.

Figure 9 shows the phase of the input, output, and impulse response. The results indicate that the phases of the output pulse are similar with the input one except for a steady phase shift, which is the phase of the impulse response. Figures $9 a, b$ shows that the phase response of the pump and Stokes pulse are, respectively, of -0.69 and $-0.75 \mathrm{rad}$ and increase slightly with light wave number. These are mainly caused by the optical distance between the incident plane and the probe center. In one word, the amplitudes of the pump and the Stokes field increase greatly, and the phase response of each spectral component is rarely disturbed.

Figures 8 and 9 show the response of the TERS nanostructure to the shaped pump and Stokes pulse. According to Eq. (1), the Raman transition probabilities of molecules in air and TERS nanostructures are calculated as a function of the wavenumber. Compared with the case without the TERS nanostructure, Fig. 10 shows that the probability of the $1520 \mathrm{~cm}^{-1}$ and $1600 \mathrm{~cm}^{-1}$ Raman transition increases by more than 4 orders of magnitude. This is because the electric field intensity at the gap center increases greatly, and the probability of Raman transition is proportional to the fourth power of the electric field intensity

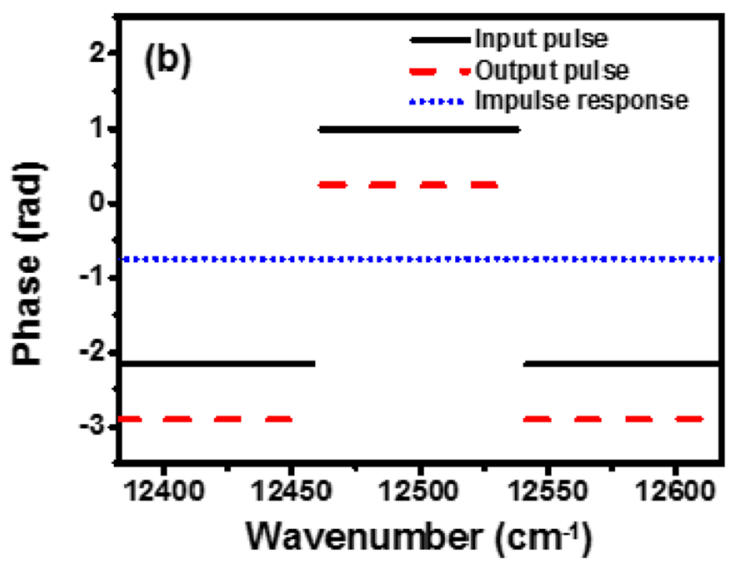

Fig. 9 Schematic diagram of the phases of the input, the output, and the impulse response of a the pump field and $\mathbf{b}$ the Stokes field 

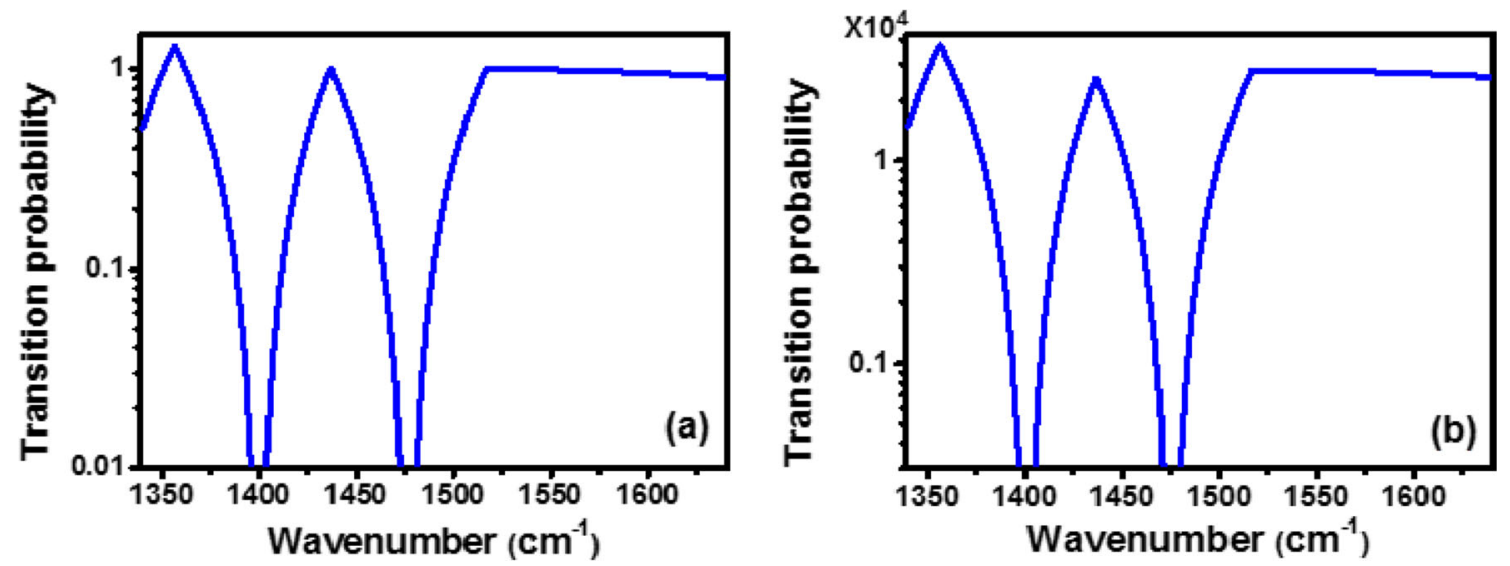

Fig. 10 The stimulated Raman transition probability $\mathbf{a}$ in air and $\mathbf{b}$ in TERS for the phase step position at $13883.3 \mathrm{~cm}^{-1}$. The probability is normalized with the value at $1520 \mathrm{~cm}^{-1}$ in air
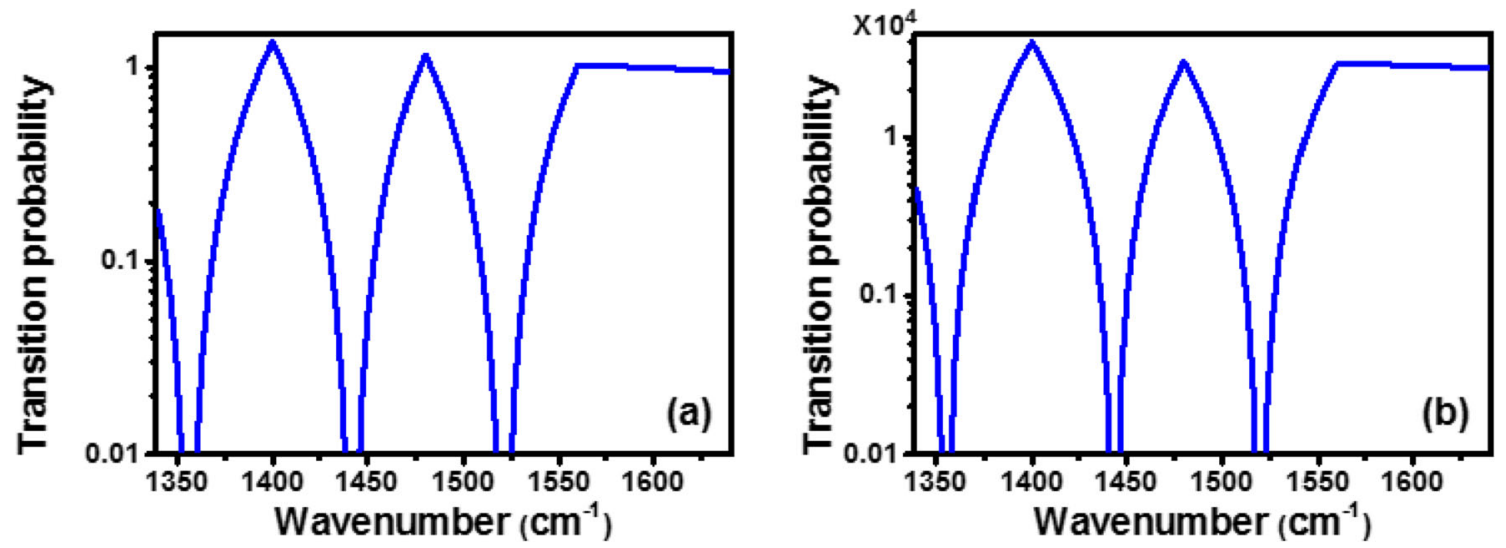

Fig. 11 The stimulated Raman transition probability $\mathbf{a}$ in air and $\mathbf{b}$ in TERS for the phase step position at $13926.7 \mathrm{~cm}^{-1}$. The probability is normalized with the value at $1600 \mathrm{~cm}^{-1}$ in air

$[44,45]$. At the same time, the transition probability in the range of 1397 to $1406 \mathrm{~cm}^{-1}$ is less than $1 \%$ of the value at $1520 \mathrm{~cm}^{-1}$. These results indicate that the enhanced and selected Raman transition is realized simultaneously, and the width of the depressed Raman peak is larger than $9 \mathrm{~cm}^{-1}$.

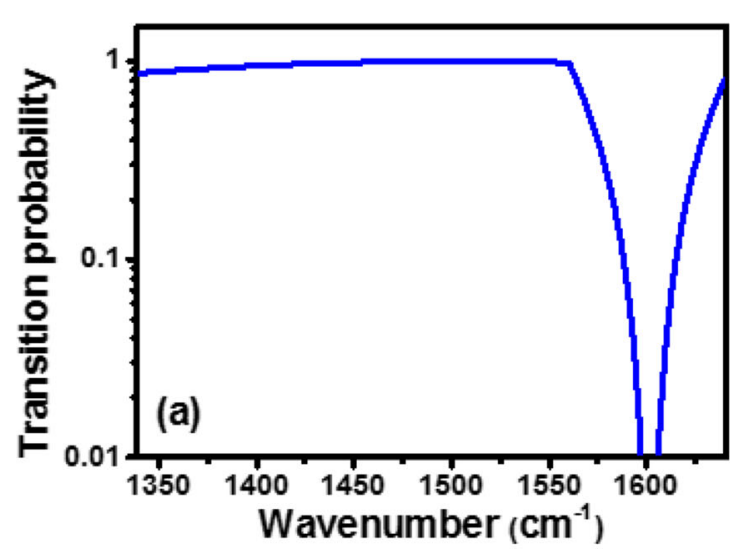

Figure 5 shows that as step position scans to $13,926.7 \mathrm{~cm}^{-1}$ and $14,166.7 \mathrm{~cm}^{-1}$, the transition probabilities of $\mathrm{pf} 2$ and $\mathrm{pf} 1$ are suppressed to zero, respectively. We study the temporal distributions of the shaped pump and Stokes pulse and the responses of the TERS nanostructures, and obtain the

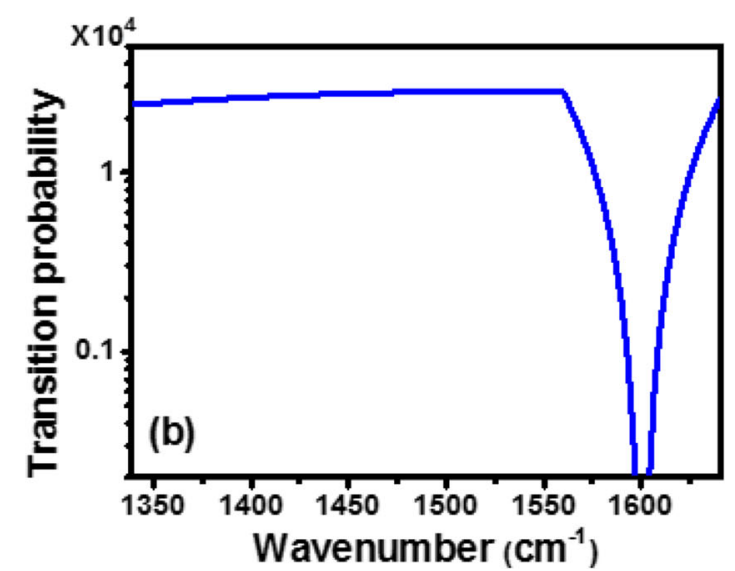

Fig. 12 The stimulated Raman transition probability $\mathbf{a}$ in air and $\mathbf{b}$ in TERS for the phase step position at $14166.7 \mathrm{~cm}^{-1}$. The probability is normalized with the value at $1520 \mathrm{~cm}^{-1}$ in air 
Raman transition probability as shown in Figs. 11 and 12. When the step position scans to $13,926.7 \mathrm{~cm}^{-1}$, the curve profiles in air and in TERS are similar, but the probability increases by $2.8 \times 10^{4}$ times of amplitude. At the same time, the Raman probability in the range of 1515 to $1524 \mathrm{~cm}^{-1}$ is less than $1 \%$ of the value at $1600 \mathrm{~cm}^{-1}$. When the step position scans to $14,166.7 \mathrm{~cm}^{-1}$, the Raman probability increases by $2.8 \times 10^{4}$ times of amplitude, and the values in the range of 1596 to $1604 \mathrm{~cm}^{-1}$ are less than $1 \%$ of the value at $1520 \mathrm{~cm}^{-1}$. In one word, the enhanced and selective Raman transition is realized in the TERS nanostructure simultaneously.

In this paper, the stimulated Raman transition is a two-step process: absorbing a photon of pump laser and emitting immediately a photon with same energy of Stokes laser. This is a non-linear interaction of pump and Stokes laser with the molecules. Recently, P. Dawson et al. reported the effects of nonlinear response of metal dielectric constant on the tipenhanced Raman scattering [48-50]. In the region around the gap of tip-substrate, the light field is greatly enhanced and a Kerr type non-linearity, locally modified dielectric constant is used to study the Raman scattering. This paper focuses on the enhanced selective Raman transition of TERS nanostructure. The effects of the non-linear locally modified dielectric constant of metal on the Raman transition are not enclosed.

\section{Conclusions}

In summary, we theoretically demonstrate a scheme for achieving precise control of selective excitation and greatly enhanced Raman transition among three excited states via TERS structure. The frequency components in the middle part of Stokes spectrum are rectangular $\pi$ phase modulated, and the others are cutoff. By $\pi$ phase scanning the pump pulses, the stimulated Raman transition probability for one excited state is suppressed to zero, while those for the other two excited states keep at the maximal value.

The shaped pump and Stokes pulse irradiate obliquely into the TERS structure. The impulse responses in temporal and frequency domain are calculated by using FDTD simulation followed by Fourier transform. The results indicate that the frequency components and the relative phase are nearly same with the corresponding input pulses, but the intensities are enhanced by more than ten times. Compared with the case without the TERS nanostructure, the probability of selective excited Raman transition increases by more than 4 orders of magnitude, and the selective depressed Raman peak keeps at 0 . The enhanced and selective Raman excitation is realized in the TERS nanostructure simultaneously, which could contribute to the enhancement of the signal-noise ratio for the Raman spectra of single molecule and its application in chemistry, biology, medicine, pharmacology, and environmental science.

Funding Information This work is supported by the National Natural Science Foundation of China (grant no. 11474097, 11374099, 11274116), the Open Fund of the State Key Laboratory of High Field Laser Physics (Shanghai Institute of Optics and Fine Mechanics), Shandong Provincial Major Science and Technology Special Project (New Emergent Industries) (2015ZDXX0801A03), Shandong Provincial Key Research and Development Project (2017CXGC0810), and Shandong Education Science Plan "Special Subject for Scientific Research of Educational Admission Examination" (ZK1337212B008).

Open Access This article is distributed under the terms of the Creative Commons Attribution 4.0 International License (http:// creativecommons.org/licenses/by/4.0/), which permits unrestricted use, distribution, and reproduction in any medium, provided you give appropriate credit to the original author(s) and the source, provide a link to the Creative Commons license, and indicate if changes were made.

\section{References}

1. Mauser N, Hartschuh A (2014) Tip-enhanced near-field optical microscopy. Chem Soc Rev 43:1248-1262

2. Arroyo JO, Kukura P (2016) Non-fluorescent schemes for singlemolecule detection, imaging and spectroscopy. Nat Photonics 10: $11-17$

3. Yu L, Hu D, Lin P, Gao F, Zhang Z, Jinglei D (2016) Optoplasmonic probe to realize scanning nearfield Raman microscopy. Opt Express 24(5):5243-5252

4. Meng L, Sun M, Chen J, Yang Z (2016) A nanoplasmonic strategy for precision in-situ measurements of tip-enhanced Raman and fluorescence spectroscopy. Sci Rep 6:19558

5. Vantasin S, Tanaka Y, Uemura S, Suzuki T, Kutsuma Y, Doujima D, Kanekod T, Ozaki Y (2015) Characterization of SiC-grown epitaxial graphene microislands using tip-enhanced Raman spectroscopy. Phys Chem Chem Phys 17:28993-28999

6. Duan S, Tian G, Luo Y (2016) Visualization of vibrational modes in real space by tip-enhanced non-resonant Raman spectroscopy. Angew Chem Int Ed 55:1041-1045

7. Klingsporn JM, Jiang N, Pozzi EA, Sonntag MD, Chulhai D, Seideman T, Jensen L, Hersam MC, Van Duyne RP (2014) Intramolecular insight into adsorbate-substrate interactions via low-temperature, ultrahigh-vacuum tip-enhanced Raman spectroscopy. J Am Chem Soc 136:3881-3887

8. Chen C, Hayazawa N, Kawata S (2014) A 1.7 nm resolution chemical analysis of carbon nanotubes by tip-enhanced Raman imaging in the ambient. Nat Commun 5:3312

9. Yano T-a, Ichimura T, Kuwahara S, H'Dhili F, Uetsuki K, Okuno Y, Verma P, Kawata S (2013) Tip-enhanced nano-Raman analytical imaging of locally induced strain distribution in carbon nanotubes. Nat Commun 4:2592

10. Zhang R, Zhang Y, Dong ZC, Jiang S, Zhang C, Chen LG, Zhang L, Liao Y, Aizpurua J, Luo Y, Yang JL, Hou JG (2013) Chemical mapping of a single molecule by plasmon-enhanced Raman scattering. Nature 498(6):82-86

11. Steidtner J, Pettinger B (2008) Tip-enhanced Raman spectroscopy and microscopy on single dye molecules with $15 \mathrm{~nm}$ resolution. Phys Rev Lett 100(6):236101

12. Kneipp K, Yang W, Kneipp H, Perelman LT, Itzkan I, Dasari RR, Feld MS (1997) Single molecule detection using surface- 
enhanced Raman scattering (SERS). Phys Rev Lett 78(9): $1667-1670$

13. Liu Y, Li X, Wu H, Zeng Z, Zhang D, Wang P, Zhang L, Fang Y (2017) Investigation of tip-enhanced Raman spectroscopy on a silver nanohole array substrate. Plasmonics 12:1861-1867

14. Dab C, Kolhatkar G, Plathier J, Thomas R, Ruediger A (2018) Dependence of apertureless scanning near-field spectroscopy on nanoscale refractive index changes. Plasmonics 13:99-106

15. Kurouski D, Mattei M, Van Duyne RP (2015) Probing redox reactions at the nanoscale with electrochemical tip-enhanced Raman spectroscopy. Nano Lett 15:7956-7962

16. Park K-D, Muller EA, Kravtsov V, Sass PM, Dreyer J, Atkin JM, Raschke MB (2016) Variable-temperature tip-enhanced Raman spectroscopy of single-molecule fluctuations and dynamics. Nano Lett 16:479-487

17. Gawinkowski S, Pszona M, Gorski A, Niedziółka-Jönsson J, Kamińska I, Nogalaa W, Waluk J (2016) Single molecule Raman spectra of porphycene isotopologues. Nanoscale 8: 3337-3349

18. Fang Y, Zhang Z, Sun M (2016) High vacuum tip-enhanced Raman spectroscope based on a scanning tunneling microscope. Rev Sci Instrum 87:033104

19. Oron D, Dudovich N, Yelin D, Silberberg Y (2002) Narrow-band coherent anti-Stokes Raman signals from broad-band pulses. Phys Rev Lett 88(6):063004

20. Oron D, Dudovich N, Yelin D, Silberberg Y (2002) Quantum control of coherent anti-Stokes Raman processes. Phys Rev A 65: 043408

21. Konradi J, Singh AK, Scaria AV, Materny A (2006) Selective spectral filtering of molecular modes of $\beta$-carotene in solution using optimal control in four-wave-mixing spectroscopy. J Raman Spectrosc 37:697-704

22. Pearson BJ, White JL, Weinacht TC, Bucksbaum PH (2001) Coherent control using adaptive learning algorithms. Phys Rev A 63:063412

23. Zhang S, Liang Z, Zhang X, Ding L, Chen G, Sun Z, Wang Z (2007) Selective excitation of CARS by adaptive pulse shaping based on genetic algorithm. Chem Phys Lett 433:416-421

24. Wu R-B, Brif C, James MR, Rabitz H (2015) Limits of optimal control yields achievable with quantum controllers. Phys Rev A 91: 042327

25. Gao F, Rey-de-Castro R, Donovan AM, Xu J, Wang Y, Rabitz H, Shuang F (2014) Pathway dynamics in the optimal quantum control of rubidium: cooperation and competition. Phys Rev A 89:023416

26. Gao F, Wang Y, Rey-de-Castro R, Rabitz H, Shuang F (2015) Quantum control and pathway manipulation in rubidium. Phys Rev A 92:033423

27. Knutsen KP, Johnson JC, Miller AE, Petersen PB, Saykally RJ (2004) High spectral resolution multiplex CARS spectroscopy using chirped pulses. Chem Phys Lett 387:436-441

28. Tok RU, Sendur K (2011) Femtosecond pulse shaping using plasmonic snowflake nanoantennas. Phys Rev A 84:033847

29. Stockman MI, Bergman DJ, Kobayashi T (2004) Coherent control of nanoscale localization of ultrafast optical excitation in nanosystems. Phys Rev B 69:054202

30. Stockman MI, Faleev SV, Bergman DJ (2002) Coherent control of femtosecond energy localization in nanosystems. Phys Rev Lett 88(6):067402

31. Lee T-W, Gray SK (2005) Controlled spatiotemporal excitation of metal nanoparticles with picosecond optical pulses. Phys Rev B 71: 035423

32. Huang JS, Voronine DV, Tuchscherer P, Brixner T, Hecht B (2009) Deterministic spatiotemporal control of optical fields in nanoantennas and plasmonic circuits. Phys Rev B 79:195441
33. Shah RA, Scherer NF, Pelton M, Gray SK (2013) Ultrafast reversal of a Fano resonance in a plasmon-exciton system. Phys Rev B 88: 075411

34. Mittal R, Glenn R, Saytashev I, Lozovoy VV, Dantus M (2015) Femtosecond nanoplasmonic dephasing of individual silver nanoparticles and small clusters. J Phys Chem Lett 6:1638-1644

35. Yang Y-Y, Scrinzi A, Husakou A, Li Q-G, Stebbings SL, Süßmann F, Yu H-J, Kim S, Rühl E, Herrmann J, Lin X-C, Kling MF (2013) High-harmonic and single attosecond pulse generation using plasmonic field enhancement in ordered arrays of gold nanoparticles with chirped laser pulses. Opt Express 21(2):2195-2205

36. Utikal T, Stockman MI, Heberle AP, Lippitz M, Giessen H (2010) All-optical control of the ultrafast dynamics of a hybrid plasmonic system. Phys Rev Lett 104:113903

37. Frontiera RR, Gruenke NL, Van Duyne RP (2012) Fano-like resonances arising from long-lived molecule-plasmon interactions in colloidal nanoantennas. Nano Lett 12:5989-5994

38. Crampton KT, Zeytunyan A, Fast AS, Ladani FT, AlfonsoGarcia A, Banik M, Yampolsky S, Fishman DA, Potma EO, Ara Apkarian V (2016) Ultrafast coherent Raman scattering at plasmonic nanojunctions. J Phys Chem C 120:20943-20953

39. Yampolsky S, Fishman DA, Dey S, Hulkko E, Banik M, Potma EO, Apkarian VA (2014) Seeing a single molecule vibrate through timeresolved coherent anti-Stokes Raman scattering. Nat Photonics 8: 650-656

40. Johnson PB, Christy RW (1972) Optical constants of the noble metals. Phys Rev B 6(12):4370-4379

41. Najjar S, Talaga D, Schué L, Coffinier Y, Szunerits S, Boukherroub R, Servant L, Rodriguez V, Bonhommeau S (2014) Tip-enhanced Raman spectroscopy of combed double-stranded DNA bundles. J Phys Chem C 118:1174-1181

42. Simmons PD Jr, Turley HK, Silverstein DW, Jensen L, Camden JP (2015) Surface-enhanced spectroscopy for higher-order light scattering: a combined experimental and theoretical study of second hyper-Raman scattering. J Phys Chem Lett 6:5067-5071

43. Jiang S, Zhang Y, Zhang R, Hu C, Liao M, Luo Y, Yang J, Dong Z, Hou JG (2015) Distinguishing adjacent molecules on a surface using plasmon-enhanced Raman scattering. Nat Nanotechnol 10: $865-870$

44. Zhang S, Shi J, Zhang H, Jia T, Wang Z, Sun Z (2010) Precise control of state-selective excitation in stimulated Raman scattering. Phys Rev A 82:043841

45. Meshulach D, Silberberg Y (1999) Coherent quantum control of multiphoton transitions by shaped ultrashort optical pulses. Phys Rev A 60(2):1287-1292

46. Cheng W, Zhang S, Jia T, Ma J, Feng D, Sun Z (2013) Coherent control of two-photon absorption of $\mathrm{Pr}^{3+}$ ion by shaped ultrashort laser pulses. Chin Opt Lett 11(4):041903

47. Ropers C, Park DJ, Stibenz G, Steinmeyer G, Kim J, Kim DS, Lienau C (2005) Femtosecond light transmission and subradiant damping in plasmonic crystals. Phys Rev Lett 94:113901

48. Dawson P, Frey D, Kalathingal V, Mehfuz R, Mitra J (2017) Novel routes to electromagnetic enhancement and its characterisation in surface- and tip-enhanced Raman scattering. Faraday Discuss 205: $121-148$

49. Aizpurua J, Arnolds H, Baumberg J et al (2017) Ultrasensitive and towards single molecule SERS: general discussion. Faraday Discuss 205:291-330

50. Graham D, Goodacre R, Arnolds H et al (2017) Theory of SERS enhancement: general discussion. Faraday Discuss 205:173-211 